\title{
RESEARCH
}

Open Access

\section{Single-cell genomics of co-sorted}

\section{Nanoarchaeota suggests novel putative host associations and diversification of proteins involved in symbiosis}

Jessica K. Jarett ${ }^{1}$ (D), Stephen Nayfach', Mircea Podar²,3, William Inskeep ${ }^{4}$, Natalia N. Ivanova', Jacob Munson-McGee ${ }^{5}$, Frederik Schulz ${ }^{1}$, Mark Young ${ }^{6}$, Zackary J. Jay ${ }^{4}$, Jacob P. Beam ${ }^{4,7}$, Nikos C. Kyrpides ${ }^{1}$, Rex R. Malmstrom¹ (B, Ramunas Stepanauskas ${ }^{7}$ and Tanja Woyke ${ }^{1 *}$ (D)

\begin{abstract}
Background: Nanoarchaeota are obligate symbionts of other Archaea first discovered 16 years ago, yet little is known about this largely uncultivated taxon. While Nanoarchaeota diversity has been detected in a variety of habitats using $16 \mathrm{~S}$ rRNA gene surveys, genome sequences have been available for only three Nanoarchaeota and their hosts. The host range and adaptation of Nanoarchaeota to a wide range of environmental conditions has thus largely remained elusive. Single-cell genomics is an ideal approach to address these questions as Nanoarchaeota can be isolated while still attached to putative hosts, enabling the exploration of cell-cell interactions and fine-scale genomic diversity.

Results: From 22 single amplified genomes (SAGs) from three hot springs in Yellowstone National Park, we derived a genome-based phylogeny of the phylum Nanoarchaeota, linking it to global 16S rRNA gene diversity. By exploiting sequencing of co-sorted tightly attached cells, we associated Nanoarchaeota with 6 novel putative hosts, 2 of which were found in multiple SAGs, and showed that the same host species may associate with multiple species of Nanoarchaeota. Comparison of single nucleotide polymorphisms (SNPs) within a population of Nanoarchaeota SAGs indicated that Nanoarchaeota attached to a single host cell in situ are likely clonal. In addition to an overall pattern of purifying selection, we found significantly higher densities of non-synonymous SNPs in hypothetical cell surface proteins, as compared to other functional categories. Genes implicated in interactions in other obligate microbemicrobe symbioses, including those encoding a cytochrome bd-I ubiquinol oxidase and a FlaJ/TadC homologue possibly involved in type IV pili production, also had relatively high densities of non-synonymous SNPS.

Conclusions: This population genetics study of Nanoarchaeota greatly expands the known potential host range of the phylum and hints at what genes may be involved in adaptation to diverse environments or different hosts. We provide the first evidence that Nanoarchaeota cells attached to the same host cell are clonal and propose a hypothesis for how clonality may occur despite diverse symbiont populations.
\end{abstract}

Keywords: Nanoarchaeota, Single-cell genomics, Symbiosis, Single nucleotide polymorphisms

\footnotetext{
* Correspondence: twoyke@lbl.gov

'DOE Joint Genome Institute, Walnut Creek, CA, USA

Full list of author information is available at the end of the article
}

(C) The Author(s). 2018 Open Access This article is distributed under the terms of the Creative Commons Attribution 4.0 International License (http://creativecommons.org/licenses/by/4.0/), which permits unrestricted use, distribution, and reproduction in any medium, provided you give appropriate credit to the original author(s) and the source, provide a link to the Creative Commons license, and indicate if changes were made. The Creative Commons Public Domain Dedication waiver (http://creativecommons.org/publicdomain/zero/1.0/) applies to the data made available in this article, unless otherwise stated. 


\section{Background}

Nanoarchaeota were first reported in 2002 when Huber and colleagues cultured Nanoarchaeum equitans, along with its host Ignicoccus hospitalis, from a marine hydrothermal vent [1]; this enabled detailed physiological, ultrastructural, and genomic studies of this unique symbiosis [2-9]. N. equitans is an obligate symbiont with a reduced genome [3], attaching to and relying on its host for almost all essential cellular components including amino acids, nucleotides, lipids, and co-factors, which appear to be trafficked via a direct cytoplasmic connection between the cells $[2,4,10]$. Later surveys of $16 \mathrm{~S}$ rRNA sequences revealed Nanoarchaeota living in diverse environments, including marine hydrothermal sediments, terrestrial hot springs in Asia, North America, and New Zealand [11-15], and mesophilic hypersaline environments [11]. Nanoarchaeota sequences were also retrieved from cool photic regions of the Yellowstone Lake, although the organisms likely originated from submerged thermal springs [15]. These environments span a variety of temperatures from 4 to greater than $100{ }^{\circ} \mathrm{C}$, and $\mathrm{pH}$ values from 3.5 to 8.0 , suggesting that Nanoarchaeota genomes may be similarly diverse. At spatial scales from a few kilometers to tens of meters, hundreds of different Nanoarchaeota OTUs have been recovered [15], some with less than 90\% identity to other known Nanoarchaeota 16S rRNA sequences [12]. However, $N$. equitans is currently the sole sequenced representative from a marine environment [3], and only two other genomes are available (Nanopusillus acidilobi and Nst1, "Nanobsidianus stetteri"), both from hot springs in Yellowstone National Park (YNP) [16, 17].

Phylogenetically, Nanoarchaeota are grouped basally in the archaeal tree of life [3] with other lineages of ultra-small Archaea termed DPANN (comprising Diapherotrities, Parvarchaea, Aenigmarchaeota, Nanohaloarchaeota, and Nanoarchaeota) [18, 19]. A number of additional phyla have been added to this group more recently (Woesearchaeota, Pacearchaeota, Micrarchaeota). Although the relationship of DPANN to other archaeal phyla remains somewhat uncertain $[19,20]$, they share many characteristics, including small genomes, limited metabolic capability, and likely a symbiotic lifestyle [21-23]. Currently available genomic data support a symbiotic common ancestor of marine and terrestrial Nanoarchaeota lineages and an ancient divergence of these two groups [17], but it is not known if the common ancestor of DPANN or of all Nanoarchaeota was symbiotic.

Initial $16 \mathrm{~S}$ rRNA surveys revealed that many habitats harboring Nanoarchaeota do not contain any of the known hosts, suggesting additional species can serve as hosts $[11,13,14]$. Only three host-symbiont pairs are known: I. hospitalis and N. equitans, Acd1 "Acidicryptum nanophilum" and "Nanobsidianus stetteri" [17], and Acidilobus 7A and Nanopusillus acidilobi [16]. Co-occurrence and other analyses have suggested additional hosts (e.g., Vulcanisaeta, Pyrobaculum [24]), and from the wide range of temperature, $\mathrm{pH}$, and physiochemical parameters described in Nanoarchaeota habitats, it is likely that there are multiple hosts. It is unclear when the radiation of terrestrial Nanoarchaeota to different environments and hosts occurred and if any of them have switched their hosts. It is also unknown whether the same species of Nanoarchaeota can associate with multiple hosts, or vice versa. Attempts to co-culture N. equitans and N. acidilobi with different hosts have not been successful $[5,16]$. Finally, while host cells with multiple Nanoarchaeota attached are frequently observed, we do not know whether genomes of these symbionts associated with a single host are clonal or heterogeneous.

A substantial body of work has been amassed with $N$. equitans and I. hospitalis, characterizing in detail their genomic, transcriptomic, proteomic, metabolomic, and ultrastructural interactions [2, 3, 5-8]. When in co-culture with $N$. equitans, I. hospitalis reduces the diversity of metabolic precursors, channels more of its energy production towards supporting the symbiont, supplies it with specific amino acid precursors, and perhaps re-routes $\mathrm{NADH}$ oxidation pathways to enhance ATP synthesis in $N$. equitans $[6,8]$. Even a single attached $N$. equitans cell retards the growth of its host in co-culture, and as they proliferate to densities of $>10$ attached cells, $N$. equitans prevents host replication altogether [5]. Further, the exponential and stationary growth phases are out of sync in $N$. equitans and I. hospitalis, with $N$. equitans continuing to grow as its host enters stationary phase [5]. Less physiological detail is known for terrestrial Nanoarchaeota, but several lines of evidence suggest that they may have fewer deleterious effects or could even be beneficial to their hosts under some conditions. Terrestrial Nanoarchaeota have slightly larger genomes than $N$. equitans and a larger repertoire of enzymes involved in carbohydrate metabolism. The overall growth of the host Acidilobus sp. 7A is not affected by co-culture with $N$. acidilobi, about half of the host cells do not have any attached $N$. acidilobi in stationary phase [5, 16], and growth kinetics are synchronized in N. acidilobi and Acidilobus sp. 7A [16]. A comparable ectosymbiosis has been observed between Actinomyces odontolyticus and a human oral member of Saccharibacteria (candidate division TM7), in which the Saccharibacteria are obligate symbionts with high host specificity, but the Actinomyces host can live independently [25]. Interestingly, while that oral Saccharibacteria behaves as a parasite in most culture conditions, they may be able to disguise or protect their hosts from human immune cells, thus acting as mutualists in a different ecological context [25]. Environmental conditions and the presence of competing organisms may be similarly important in understanding the full range of interactions between Nanoarchaeota and their hosts. 
Nanoarchaeota share some similarities with other known obligate microbial symbionts (e.g., insect endosymbionts), including reduced genomes and reliance on a host [26], but have important differences that may lead to different evolutionary pressures and trajectories [27]. As ectosymbionts, Nanoarchaeota have access to external sources of DNA including lateral gene transfer with their hosts [7] and with other Nanoarchaeota via viral transduction [28, 29]. They have also retained a full suite of genes for DNA recombination and repair $[3,16,17]$, and RNA-Seq data from $N$. equitans [30] suggests that genome fragmentation, inversion, re-arrangement, and splitting of protein-coding genes $[3,17,28]$ are ongoing processes in Nanoarchaeota genomes. Together with large population sizes [28], these factors likely prevent the bottlenecks and genetic drift that degrade the genomes of many endosymbionts [31-35]. With multiple genomes available, mapping of single nucleotide polymorphisms (SNPs) can be used to compare selective pressures on different genes [36, 37] and, together with comparisons of gene repertoire, may show how Nanoarchaeota have specialized to different hosts or environmental niches.

In this study, we have analyzed 22 Nanoarchaeota SAGs from three hot springs in YNP; some of these Nanoarchaeota were co-sorted with their putative hosts, allowing us to suggest expansions to host range. We leveraged single-cell genomics of these co-sorted cells [38] to investigate the diversity of Nanoarchaeota on a single host cell. Lastly, we performed SNP analysis to look at patterns of selection within functional categories of genes, using diversification as a signature for proteins potentially involved in symbiosis. Exploring the functions of these proteins allowed us to draw new parallels between terrestrial and marine Nanoarchaeota, and with other microbe-microbe symbioses.

\section{Methods}

\section{Single-cell sequencing and SAG binning}

Hot spring sediment samples for single-cell genomics were collected from Cistern Spring and Echinus Geyser hot springs in YNP in 2011 (Additional file 1: Figure S1) [39]. Cells were separated from sediment, concentrated using Nycodenz density gradient centrifugation, and frozen on dry ice. Single cells were isolated using fluorescent-activated cell sorting (FACS), lysed and whole genome amplified with multiple displacement amplification (MDA), and MDA products were screened with $16 \mathrm{~S}$ rRNA gene PCR according to DOE JGI standard protocols [40]. Based on 16S rRNA gene sequences, 6 Nanoarchaeota cells were selected, 2 from Cistern Spring and 4 from Echinus Geyser. Nextera libraries with a target insert size of 300 were sequenced on the Illumina NextSeq platform following the standard Illumina TruSeq protocol (Illumina) generating between 12,722,302 and 23,436,168 reads per SAG (Additional file 2: Table S1). Adapters were trimmed from the sequence data, reads were filtered for quality, errors were corrected with tadpole, and a kmer normalization was performed using bbnorm; the latter two steps were performed with the bbtools package [41]. Filtered reads were then assembled with SPAdes version 3.10 .1 [42] with kmer sizes of 25, 55, and 95; resulting scaffolds were trimmed by $200 \mathrm{bp}$ on each end, and trimmed scaffolds greater than $2 \mathrm{~kb}$ in length were retained.

We discovered after sequencing that some of the SAGs contained both Nanoarchaeota and putative host genomes, and therefore are not technically single amplified genomes. However, these do represent single sorting events, so for the sake of simplicity, we will refer to all MDA products originating from a single sorting event as SAGs. We use "single-sort" to refer to SAGs containing only Nanoarchaeota sequence and "co-sort" to refer to SAGs containing both Nanoarchaeota and putative host sequence. Bins derived from co-sort SAGs are referred to as genome bins. Co-sorted SAGs were also detected among 16 recently generated SAGs of Nanoarchaeota from Nymph Lake in YNP, so these were added to the analysis to expand the survey of putative host range and environments [28, 29]. To separate scaffolds originating from Nanoarchaeota and putative hosts within the 6 SAGs from this study and the 16 SAGs from Nymph Lake (Additional file 2: Table S1; Additional file 1: Figure S1), scaffolds were binned using MetaBAT [43] with default settings and a minimum bin size of $50 \mathrm{~kb}$, then bins were manually refined in Anvi'o [44] based on GC content and BLAST [45] comparison to NCBI nr. Standard assembly statistics, completeness, and redundancy of bins were assessed with CheckM [46]. Tetranucleotide frequencies (TNF) were calculated for scaffolds, clustered with principal components analysis (PCA), and plotted in $\mathrm{R}$ to visually check binning results.

Genome bins were assigned as putative hosts or Nanoarchaeota based on their GC content, the lineage assigned by CheckM, TNF PCA plots, and average nucleotide identity (ANI) to reference genomes of hosts and Nanoarchaeota. ANI analysis was performed with pyani with -m ANIb $[47,48]$ and visualized with the superheat package [49] in R. Genome bins were filtered by different criteria for different analyses (Additional file 2: Table S1). Nanoarchaeota or putative host genome bins had to be at least $25 \mathrm{~kb}$ in size for inclusion in heatmaps, and both bins had to be at least $25 \mathrm{~kb}$ for associating Nanoarchaeota with putative hosts. For inclusion in the ribosomal protein-based phylogeny, at least $20 \%$ of the sites in the concatenated alignment had to contain information, equivalent to approximately six ribosomal proteins.

\section{Delineation of Nanoarchaeota clades}

To delineate clades within the Nanoarchaeota, a 3-pronged approach was used: $16 \mathrm{~S}$ rRNA gene similarity, ribosomal protein-based (RP) phylogeny, and ANI. At least two of the 
following three criteria had to be met in order for genomes or genome bins to be grouped together into a clade: they had to share at least 98\% 16S rRNA gene similarity [50], be each other's nearest neighbors in the RP tree or be part of a branch containing only members of the same clade, or share at least 95\% ANI over at least $20 \mathrm{~kb}$ of alignment length. Once initial clades were formed, additional genome bins were added to clades based on 95\% ANI, but criteria that were not met could only be due to missing data, not to conflicting data. For example, a genome bin might be placed in a clade even if it did not have a $16 \mathrm{~S}$ rRNA gene sequence but not if it had a $16 \mathrm{~S}$ rRNA sequence less than $98 \%$ similar to others in the clade. All other genomes and genome bins were left unassociated with any clade ("no clade").

A phylogeny based on the concatenated alignment of ribosomal proteins ( $\mathrm{RP})$, the $\mathrm{RP}$ tree, was constructed as described previously [51] with some modifications. Briefly, best-hit sequences from 30 ribosomal protein COGs were identified with hmmsearch (HMMER v3.1b2, [52]) and extracted from genomes and Nanoarchaeota genome bins. Three COGs were absent from all genomes (COG088, COG0091, COG0099), and 3 COGs which were represented by only 1 Nanoarchaeota genome or genome bin (COG0096, COG00197, COG0255) were not included in the concatenated alignment, for a total of 24 COGs. The species tree was calculated with PhyloBayesMPI [53] CAT+GTR in two chains with $~ 3200$ trees per chain; the first $25 \%$ of trees in each chain were discarded as burn-in and the chains converged with maxdiff $<0.1$. The final tree was visualized and annotated in $\mathrm{R}$ with ggtree [54]. Pairwise comparisons of 16S rRNA gene similarity were performed in Jalview [55].

A 16S rRNA gene phylogeny was constructed to compare these newly defined clades to the larger context of phylum Nanoarchaeota globally. 16S rRNA sequences from Nanoarchaeota genome bins and Nanoarchaeota reference genomes were identified based on annotation in IMG or by structural homology search with SSU-align [56]. All 16S rRNA gene sequences (at least $400 \mathrm{nt}$ in length) assigned to phylum Nanoarchaeota in SILVA (release 128) were verified by the search and classify feature of the online SINA aligner, comparing the query sequence to up to ten neighbors with at least $75 \%$ sequence similarity, and sequences re-assigned to phylum Nanoarchaeota were retained [57, 58]. An environmental PCR amplicon dataset and PCR amplicons from sorted single cells were also included, and Candidatus Mancarchaeum acidiphilum was selected as an outgroup for rooting the tree. Sequences were aligned with SSU-align [56], masked with the default Archaea mask, and a maximum likelihood (ML) tree was created with IQ-TREE $[59,60]$ with model $\mathrm{TN}+\mathrm{R} 3$ and 100 bootstraps. Trees were visualized and annotated in $\mathrm{R}$ with package ggtree [54], using metadata from SILVA to assign sequences to habitat types.

\section{Associating Nanoarchaeota with putative hosts}

Taxonomy was assigned to putative host genome bins by ANI comparison to other genome bins and publicly available references (Additional file 2: Tables S2 and S3), requiring an ANI of at least $95 \%$ over at least $20 \mathrm{~kb}$ alignment length. None of the putative host genome bins contained a $16 \mathrm{~S}$ rRNA gene sequence so these were not compared. Standard assembly statistics, completeness, and redundancy of references were assessed with CheckM [46] and used to annotate ANI heatmaps in $\mathrm{R}$ with the package superheat [49]. Alluvial plots showing the distribution of Nanoarchaeota clades, associated putative hosts, and sampling sites were drawn in $\mathrm{R}$ with the package alluvial [61].

To investigate further possible links between Nanoarchaeota and their putative hosts, we searched for recent horizontal gene transfer by aligning all proteins at least 100 amino acids in length from our SAGs to each other and to the NCBI nr database [62]. Alignments were sorted by bitscore to obtain the top 10 overall hits, and hits between Nanoarchaeota proteins and their putative host (or vice versa for putative host proteins) were retained. Matches were required to be from the same co-sorted SAG or from the same host-Nanoarchaeota pairing (for example, clade 2 Nanoarchaeota with Thermocladium sp.).

\section{Clonality of Nanoarchaeota associated with a single host cell}

Reads from SAGs were used to call SNPs and determine if multiple symbionts with distinct genomes were attached to the same host cell. Briefly, reads were mapped from individual SAGs to the corresponding genome bins with bowtie2 (--very-sensitive, global alignment mode) and alignments were filtered to discard reads with less than $95 \%$ identity to the assembly, average read quality of less than 30, map quality of less than 20, and bases with a quality of less than 30. Pysam was used to generate read counts of the four nucleotides at each genomic position. In order to make comparisons between SAGs, all SAGs were down-sampled to 50 mapped reads per site. SNPs were called at a minor allele frequency (MAF) of at least $10 \%$ in order to minimize the effect of sequencing errors while maintaining sensitivity to detect true SNPs. Mapping and SNP calling was performed on single-sort Nanoarchaeota SAGs and co-sorted SAGs where both genome bins were at least $25 \mathrm{~kb}$ in size, except two SAGs for which reads were not available (AB-777-F03, AB-777-O03) (Additional file 2: Table S1). The distribution of SNP density was compared between co-sorted Nanoarchaeota genome bins, single-sorted Nanoarchaeota, and putative host genome bins using a one-way Wilcoxon rank sum test. Single-sorted Nanoarchaeota and putative host genome bins were presumed to represent single cells, so their variance served as a baseline for errors introduced by MDA, sequencing, and assembly. 
A simulation was performed to estimate the expected number of SNPs that would be observed from multiple distinct Nanoarchaeota attached to the same host cell. For this analysis, we selected 14 Nanoarchaeota SAGs from Nymph Lake since these symbionts were found in the same environment and therefore are most likely to co-occur on the same host cell. AB-777-F03, the most contiguous large assembly from Nymph Lake (Table 1), was used as a reference for mapping SAG reads, using the same parameters as before. We retained 6 SAGs which covered the reference genome by $>25 \%$ (Additional file 2: Table S1). To simulate the presence of multiple attached cells, we pooled mapped reads from between 1 to 6 Nanoarchaeota SAGs and used the pooled reads to call SNPs. To equalize differences in sequencing depth, we used the same number of reads from each SAG per genomic position. Each genomic position was down-sampled to 50 mapped reads and SNPs were called at a MAF of at least $10 \%$.

\section{Population diversity of Nanoarchaeota}

We used the tool MIDAS [63] to investigate the diversity of Nanoarchaeota within a single population using the same mapping and filtering parameters as before. Specifically, we used SAG reads from clade 1 SAGs from Nymph Lake, the clade with the largest number of representatives. Reads from SAGs with clade 1 Nanoarchaeota genome bins at least $100 \mathrm{~kb}$ in size $(n=7$, Additional file 2: Table S1) were mapped against AB-777-F03, as described above. Synthetic reads were created for AB-777-O03 by shredding contigs with randomreads.sh from the bbtools package [41] with 20X coverage, insert size range of 180$400 \mathrm{nt}$, read length of $150 \mathrm{nt}$, and without simulating sequencing error. To minimize the effect of sequencing errors, we used mapped reads to call the consensus allele at each genomic position within each SAG and masked sites where $>10 \%$ of the reads differed from each other. SNPs were called at genomic positions covered by at least 5 of the 7 SAGs where at least 1 SAG had an observed variant relative to the other SAGs or the reference. Within protein coding regions, we identified SNPs at fourfold degenerate sites (i.e., synonymous SNPs or sSNPs) and SNPs at onefold degenerate sites (i.e., non-synonymous SNPs or nSNPs). As a measure of selective pressure, we computed $\mathrm{pN} / \mathrm{pS}$, defined as the ratio of the number of nSNPs per non-synonymous site to the number of sSNPs per synonymous site. SNP density was computed genome wide, for classes of SNPs and for individual genes. Genes were divided into functional categories based on their annotations in IMG and only genes with at least 100 total mapped sites were considered. SNP density was compared between functional categories with at least ten genes (excluding categories oxidative stress, secretion, and

Table 1 Assembly statistics, completeness and contamination estimates, and additional information for Nanoarchaeota genome bins

\begin{tabular}{|c|c|c|c|c|c|c|c|c|c|}
\hline Genome bin ID & $\begin{array}{l}\text { Assembly } \\
\text { size (bp) }\end{array}$ & \# Scaffolds & $\begin{array}{l}\text { Longest } \\
\text { scaffold (bp) }\end{array}$ & GC (\%) & $\begin{array}{l}\text { \# Predicted } \\
\text { genes }\end{array}$ & $\begin{array}{l}\text { Estimated } \\
\text { completeness } \\
\text { (CheckM) (\%) }\end{array}$ & $\begin{array}{l}\text { Estimated } \\
\text { contamination } \\
\text { (CheckM) (\%) }\end{array}$ & $\begin{array}{l}\text { \# Ribosomal } \\
\text { proteins } \\
\text { (of 24) }\end{array}$ & $\begin{array}{l}\text { Genome } \\
\text { quality } \\
\text { (MISAG) }\end{array}$ \\
\hline AB-777-F03 Nano & 449,376 & 20 & 95,961 & 24.5 & 527 & 54.67 & 0 & 21 & Medium \\
\hline AB-777-O03 Nano & 549,214 & 47 & 44,534 & 24.1 & 656 & 65.03 & 6.54 & 21 & Medium \\
\hline AD-903-B02 Nano & 135,497 & 24 & 20,845 & 24.5 & 184 & 16.74 & 0.47 & 1 & Low \\
\hline AD-903-B22 Nano & 218,763 & 37 & 15,769 & 24.3 & 266 & 18.54 & 0 & 6 & Low \\
\hline AD-903-D09 Nano & 28,158 & 6 & 11,989 & 25.2 & 37 & 1.25 & 0 & 1 & Low \\
\hline AD-903-D23 Nano & 101,465 & 22 & 17,152 & 25.8 & 137 & 20.91 & 0 & 11 & Low \\
\hline AD-903-F05 Nano & 125,027 & 23 & 12,408 & 25.1 & 167 & 19.63 & 0 & 7 & Low \\
\hline AD-903-F18 Nano & 56,402 & 12 & 9231 & 23.9 & 80 & 12.31 & 0 & 8 & Low \\
\hline AD-903-114 Nano & 125,613 & 25 & 10,234 & 25 & 164 & 15.29 & 0.93 & 6 & Low \\
\hline AD-903-L04 Nano & 105,782 & 15 & 17,449 & 26.1 & 126 & 10.75 & 0 & 1 & Low \\
\hline AD-903-M20 Nano & 105,795 & 21 & 12,044 & 25.1 & 139 & 19.16 & 0 & 6 & Low \\
\hline AD-903-N05 Nano & 210,845 & 37 & 19,172 & 24.9 & 249 & 26.01 & 0 & 4 & Low \\
\hline AD-903-P15 Nano & 273,481 & 40 & 24,686 & 24.3 & 336 & 34.97 & 0 & 5 & Low \\
\hline AD-903-P16 Nano & 192,530 & 37 & 14,587 & 25.7 & 248 & 29.55 & 0 & 11 & Low \\
\hline CS1 Nano & 106,721 & 18 & 14,946 & 24.1 & 136 & 13.92 & 0 & 3 & Low \\
\hline CS2 Nano & 199,134 & 39 & 16,387 & 24.2 & 245 & 26.87 & 0 & 8 & Low \\
\hline EG1 Nano & 231,923 & 36 & 31,901 & 25.4 & 277 & 17.63 & 0 & 4 & Low \\
\hline EG2 Nano & 144,926 & 23 & 15,304 & 25.1 & 179 & 22.27 & 0 & 7 & Low \\
\hline EG3 Nano & 65,600 & 12 & 10,727 & 25.6 & 80 & 9.06 & 0 & 2 & Low \\
\hline EG4 Nano & 56,920 & 15 & 12,800 & 25.6 & 80 & 8.09 & 0 & 3 & Low \\
\hline
\end{tabular}


transporters) using a one-way analysis of variance and post hoc Tukey HSD test in R. For two proteins with high nSNP densities (see the "Results and discussion" section), we tested whether the distribution of nSNPs (amino acid substitutions) between internal, external, and transmembrane regions of the proteins was significantly different with chi-square tests on alignments trimmed and divided in $\mathrm{R}$.

\section{Results and discussion}

\section{Diversity and clades within Nanoarchaeota}

In this study, we utilized single-cell genomics to address ecological and evolutionary questions about Nanoarchaeota and their hosts that could not be tackled by previous studies focusing on single examples of associations. From a total pool of 22 SAGs, 4 of the 6 SAGs sequenced in this study and 6 of the 16 SAGs from Nymph Lake [28, 29] represented co-sorted SAGs with both Nanoarchaeota and putative host genome bins larger than $25 \mathrm{~kb}$ (Table 1, Additional file 2: Tables S1 and S4). Nanoarchaeota genome bins ranged from $28,158 \mathrm{bp}$ to $549,214 \mathrm{bp}$ in size (Table 1) and were clearly separated from putative host genome bins by TNF PCA in all co-sorted SAGs (Additional file 1: Figure S2). These symbiont bin sizes approximated 1 to $83 \%$ estimated genome completeness (Table 1, Additional file 2: Table S4). Although this is less than observed for single-cell genomes of benchmark cultures [64], the low estimates can be explained by low sequencing coverage of some SAGs (Additional file 1: Table S1). Additionally, the absence of some standard single-copy marker genes from Nanoarchaeota results in estimated completeness values lower than actual genome completeness. Overall, 2 Nanoarchaeota and 3 putative host genome bins met medium-quality draft MISAG standards [65], all others were low-quality drafts.

Results from ANI analysis, 16S rRNA gene similarity, and ribosomal protein phylogeny defined Nanoarchaeota groupings that were consistent for all cases where multiple metrics were available (Fig. 1, Additional file 2: Tables S5, S6, and S7). Based on these metrics, two novel approximately species-level clades of Nanoarchaeota were identified with 95-98\% 16S rRNA gene similarity and 88.7-91.8\% ANI to each other and to described species of Nanoarchaeota. These clades were used as a foundation for subsequent analyses. Clade 1 contained 9 genome bins from Nymph Lake, clade 2 contained 2 genome bins from Echinus Geyser, and 1 genome bin was associated with the previously described species Nanopusillus acidilobi. Eight genome bins could not be grouped into clades with other genome bins or references (Additional file 2: Table S1). Clades were restricted to single sampling locations, with the exception of $N$. acidilobi which we found in Nymph Lake (AD-903-F05) and was observed previously in Cistern Spring (Fig. 1). Cistern Spring,
Echinus Geyser, and Nymph Lake all harbored multiple clades of Nanoarchaeota (Fig. 1).

There are numerous members of the phylum Nanoarchaeota with 16S rRNA gene sequences that are only about $80 \%$ similar to those from sequenced genomes (Additional file 1: Figure S3); for example, $N$. equitans has $82.1 \%( \pm 0.42)$ mean $16 \mathrm{~S}$ rRNA gene similarity to other full-length sequences (Additional file 2: Table S7). Even near-identical $16 \mathrm{~S}$ rRNA sequences can accompany very different genome content $[66,67]$, thus considering only $16 \mathrm{~S}$ rRNA gene sequences can mask extensive genetic diversity and niche partitioning. The majority of available Nanoarchaeota 16S rRNA gene sequences and sequenced genomes originated from hot springs within YNP, but much of the diversity within the phylum is found in hydrothermal sediment, marine, and hypersaline habitats and is still not represented by sequenced genomes, or even full-length 16S rRNA gene sequences (Additional file 1: Figure S3). These Nanoarchaeota without genomic representation are likely to encode functional diversity critical for biogeochemical processes and evolutionary diversification of microorganisms within these ecosystems.

\section{Associating Nanoarchaeota with putative hosts}

Co-sorting of Nanoarchaeota attached to other cells has been observed previously [28], and these have been experimentally demonstrated to be host cells $[16,17]$. In this study, we expanded on this by investigating a large number of co-sorted cells from multiple sampling sites to identify novel putative hosts. Taxonomy was assigned to putative host genome bins in 9 of 10 co-sorted SAGs based on ANI to references (Additional file 1: Figure S4; Additional file 2: Table S8). Seven putative hosts were associated with Nanoarchaeota genome bins (Fig. 2), including the previously known host Acd1 "Acidicryptum nanophilum," which was observed with Nanoarchaeota clade 1 in two SAGs, lending support that the co-sorting method recovers genuine biological associations. Our data suggests that three other members of the Order Sulfolobales were hosts: Metallosphaera sp., Sulfolobus type II, and Sulfolobus sp. Thermocladium sp., Caldivirga sp., and Vulcanisaeta sp. are the first members of Order Thermoproteales implicated as possible hosts. Co-occurrence data from Kamchatka hot springs previously suggested but could not confirm Vulcanisaeta as a host [24]. Clade 2 Nanoarchaeota were found to associate with Thermocladium sp. in two SAGs. Four proteins were found to likely be horizontally transferred between Thermocladium sp. and clade 2 Nanoarchaeota (Additional file 2: Table S9). All lacked functional annotation but may provide useful information for future studies. Each Nanoarchaeota-putative host pairing was restricted to a single sampling site, although Nymph Lake and Echinus Geyser harbored multiple host-symbiont pairs (Fig. 2). 


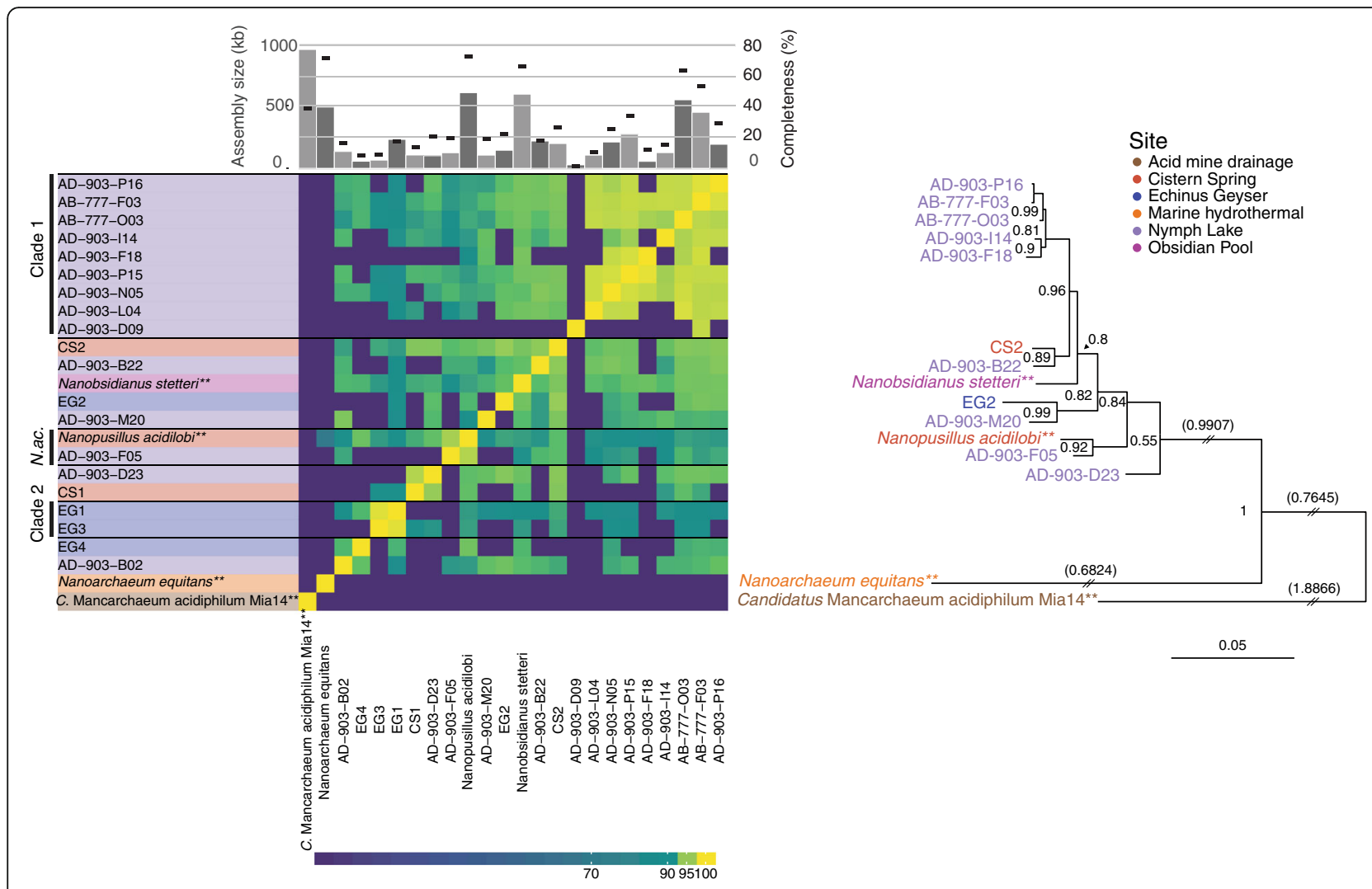

Fig. 1 Genome-based phylogeny of phylum Nanoarchaeota. Heatmap of ANI, with Bayesian phylogeny based on the concatenated alignment of ribosomal proteins. ANI comparisons with alignment lengths less than $20 \mathrm{~kb}$ were set to $0 \%$ ANI. Only genomes with information in at least $20 \%$ of aligned sites were included in the phylogenetic tree, omitting genomes with insufficient information. Species-level clades derived from a combination of ANI, 16S rRNA gene similarity, and Bayesian phylogeny are delineated by black bars beside SAG or genome names. Bar labeled "N.ac." denotes a clade formed by the cultivated species Nanopusillus acidilobi and a SAG. Clade 2 was derived from ANI and 16S rRNA gene similarity only; these genomes had insufficient ribosomal protein information for inclusion in the phylogeny. Genomes are colored by sampling location, and ** indicates a publicly available reference genome. Assembly size (bars) and estimated completeness (dashes) of Nanoarchaeota genomes are shown above the heatmap. Note that the reduced genomes of Nanoarchaeota result in estimated completeness $<100 \%$ even for closed genomes (e.g., N. equitans). Branches in the tree with a double slash have been re-scaled, and their actual branch lengths are shown in parentheses. Branch support values are indicated at tree nodes. Candidatus Mancarchaeum acidiphilum is included as an outgroup and is not part of phylum Nanoarchaeota

Notably, our data also suggest an expansion of the possible associations for the host "A. nanophilum". Previous samples from the Obsidian Pool found this host with "Nanobsidianus stetteri" [17, 24], whereas at Nymph Lake it was associated with the closely related clade 1 Nanoarchaeota (Fig. 2). This new diversity of putative host-symbiont associations raises questions about their molecular mechanisms of attachment and metabolite transfer. The physical interface between $N$. equitans and $I$. hospitalis is complex, presumably due to the unique anatomy of I. hospitalis [2, 9]. The connections between terrestrial Nanoarchaeota and their hosts have not been visualized at this level of detail, but are likely to differ substantially from those of $N$. equitans, based on what is known about the morphology and physiology of their hosts. The known and putative hosts of terrestrial Nanoarchaeota have a cell envelope consisting of an S-layer protein or proteins [68, 69], whereas in I. hospitalis the S-layer is absent [2]. Further, the detailed architecture of the $S$ layer is conserved between some putative hosts such as those within Order Sulfolobales [68]. These factors indicate that the mechanisms and structures that mediate host-symbiont interactions in terrestrial Nanoarchaeota may be more generalized or perhaps more rapidly evolving, facilitating a broader host range.

\section{Clonality of Nanoarchaeota associated with a single host cell}

Multiple Nanoarchaeota cells are commonly observed attached to a single host cell, both in culture and in situ $[5,16$, $28,70]$. However, it is not known if these cells are clonal or if the attachment of multiple, diverse Nanoarchaeota cells is possible. To assess this, we used read-mapping to quantify the density of SNPs in genome bins of attached (co-sorted) 


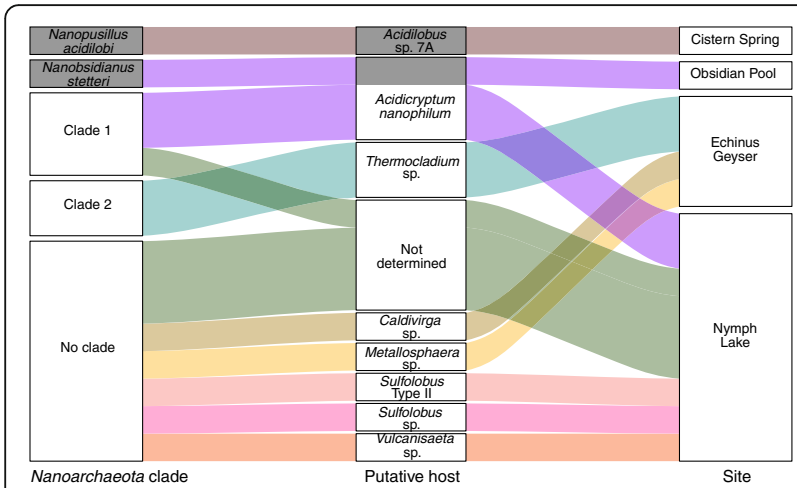

Fig. 2 Association of terrestrial Nanoarchaeota, known or putative hosts, and sampling sites. Nanoarchaeota clades are as shown in Fig. 1, including 3 SAGs from clade 1, 2 SAGs from clade 2, and 8 SAGs not assigned to a clade. ANI identification of putative hosts is shown in Additional file 1: Figure S4. Putative hosts listed as "Not determined" shared less than 95\% ANI and/or less than $20 \mathrm{~kb}$ aligned length with all other genome bins and references.

Only references and SAGs where both Nanoarchaeota and putative host genome bins were at least $25 \mathrm{~kb}$ in size are shown. Connections are colored

by putative host, and known symbioses are shaded in gray

Nanoarchaeota. As a control, we compared this to SNPs found in genome bins of Nanoarchaeota putative hosts and unattached (single-sort) Nanoarchaeota (Additional file 2: Table S1). Putative hosts were expected to be single cells, because doublets of these larger cells would have been too large to be included within the FACS gating. Likewise, single-sort Nanoarchaeota were also expected to be single cells, as has been observed in culture. Both of these groups served as a baseline for the number of SNPs to expect from a single cell. If the number of SNPs in co-sorted Nanoarchaeota was similar to this baseline, we expect that either only a single symbiont cell was attached to a host cell, or all of the symbiont cells arose from the proliferation of the same parent cell. If the number of SNPs in co-sorted Nanoarchaeota was significantly greater than the baseline, it would be an indication that there were multiple, different Nanoarchaeota attached to the same host cell.

We found no significant difference in SNP density with putative hosts (one-way Wilcoxon rank sum test, $p=0.93$ ) or with single-sort Nanoarchaeota (one-way Wilcoxon rank sum test, $p=0.62$ ) (Fig. 3a; Additional file 2: Table $\mathrm{S} 10)$. The $0.25 \mathrm{SNPs}$ per $\mathrm{kb}$ observed for co-sorted Nanoarchaeota is likely caused by a combination of errors from amplification, sequencing, and assembly [64, 71]. As an additional control, we pooled reads from multiple Nanoarchaeota SAGs to simulate diverse populations and estimate the number of SNPs expected from multiple attached cells. Here, we observed a range of 10-50 SNPs per $\mathrm{kb}$ when pooling reads from between 2 and 6 SAGs (Fig. 3b, Additional file 2: Table S11). Together, these results indicate that multiple diverse Nanoarchaeota were not attached to individual host cells-instead, there were either multiple clonal cells or only one cell per host. Although we cannot rule out the latter possibility, it seems unlikely given extensive imaging of marine and terrestrial Nanoarchaeota co-cultures [1, 5, 16] and environmental samples [28]. Nanoarchaeota are only capable of dividing while attached to a host, and often appear in clustered or linear arrangements (Additional file 1: Figure S5) that are consistent with the proliferation of a single symbiont. We suggest that future studies should leverage single-cell genomics in combination with imaging to determine the patterns of symbiont abundance on a host in situ, and further elucidate the molecular underpinnings of host establishment and the exclusivity of the symbiont in a given host.

\section{Fine-scale genomic diversity of Nanoarchaeota}

To assess genomic variation on a population scale, we analyzed the diversity within the population of clade 1 Nanoarchaeota from Nymph Lake. This was the largest species-level group of SAGs in our dataset and was sampled from the same location and date, so they represent a snapshot of diversity within this population. Genome wide, we observed an average of 28 SNPs per kb indicating significant levels of diversity within the clade. Additionally, the $\mathrm{pN} / \mathrm{pS}$ ratio of 0.159 suggests strong purifying selection and large population size. This pattern was also consistent when comparing individual SAGs to the reference genome (Additional file 1: Figure S6). There were relatively few SNPs in intergenic regions (Additional file 1: Figure S6; Additional file 2: Table S12), likely due to the high coding density in Nanoarchaeota. These patterns contrast with those of many microbial endosymbionts, which are subject to population bottlenecks, isolation, and genetic drift, eventually reaching "genomic stasis" [72-74]. Several factors act against these restrictions in Nanoarchaeota: they may be motile at some stage in their life cycle [17], they are externally attached to their hosts, and they are often abundant and diverse in situ $[12,28$, 29]. We observed considerable diversity even within this small sampling of clade 1 genome bins. Thus, we postulate that population bottlenecks are much less severe in Nanoarchaeota than in endosymbionts and that selection rather than genetic drift is primarily responsible for fixing mutations in Nanoarchaeota.

Given this pattern of purifying selection, we expected to find different densities of nSNPs across different functional categories of genes, depending on how essential the genes are and if they are involved in interaction with a host. Indeed, while there were no significant differences in the densities of SSNPs across functional categories of genes (one-way ANOVA, $F(9,297)=0.989$, $p=0.449$ ) (Additional file 2: Figure S7), some functional categories did have significantly different densities of 

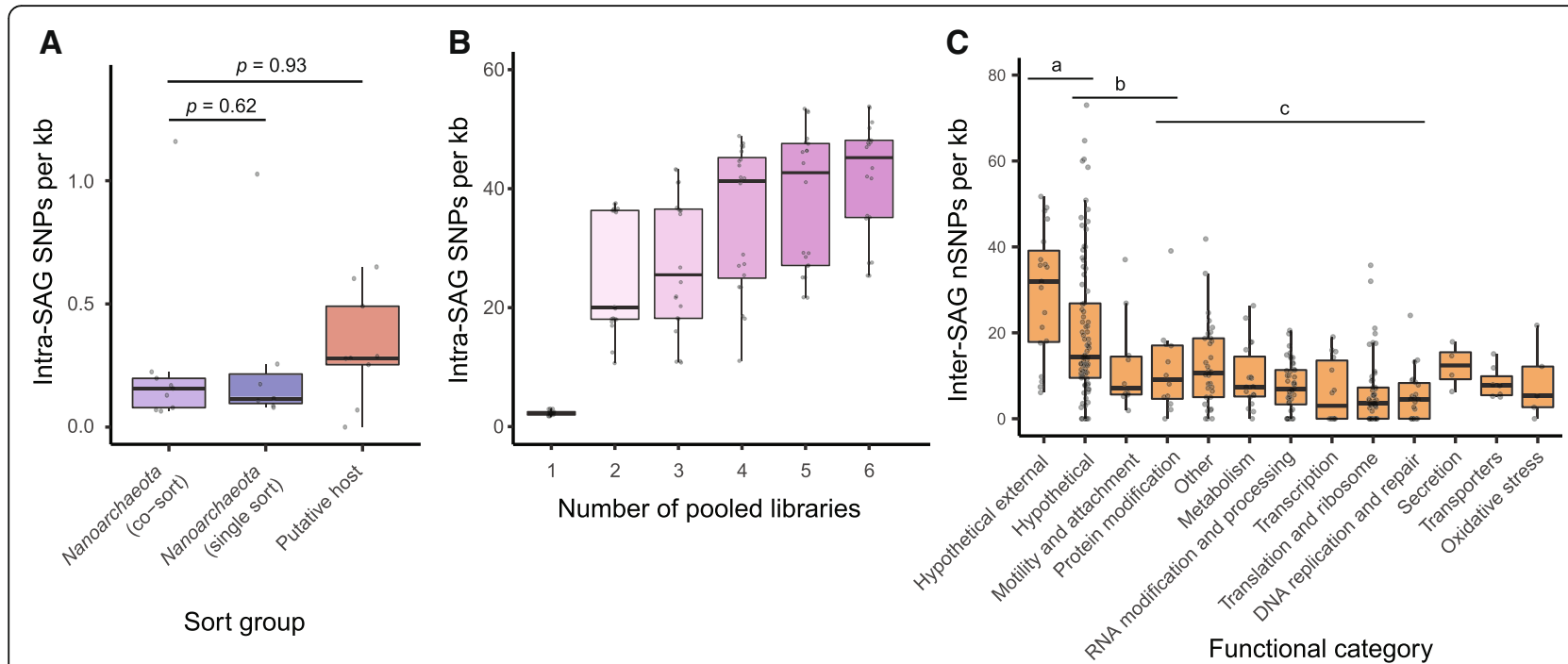

Fig. 3 Diversity of Nanoarchaeota on a single host cell and within a population. a SNPs per kb (MAF > 10\%) in single-sorted Nanoarchaeota SAGs, co-sorted Nanoarchaeota genome bins, and co-sorted putative host genome bins. There was no significant difference (one-way Wilcoxon rank sum tests) between categories. b SNPs per kb (MAF> 10\%) in pooled datasets of 1 to 6 SAGs, simulating results expected if multiple distinct Nanoarchaeota were attached to a host cell. c Variation in nSNP density in clade 1 Nanoarchaeota genes, summarized by functional category. Different letter groups have significantly different nSNP densities, from ANOVA (one-way ANOVA, $F(9,297)=12.66, p<2 \mathrm{e}-16$ ) and post hoc Tukey HSD tests $(p<0.05)$ (Additional file 2: Table S13). No bar over boxes indicates categories with fewer than ten genes which were not included in ANOVA or post hoc tests

nSNPs (one-way ANOVA, $F(9,297)=12.66, p<2 \mathrm{e}-16$ ) (Fig. 3c; Additional file 2: Table S13). Highly essential categories such as translation and ribosome proteins, DNA replication and repair, RNA modification and processing, and transcription expected to be under strong selective pressure to maintain function had low densities of nSNPs (Fig. 3c). A few categories had significantly higher densities of nSNPs, including protein modification, motility and attachment, hypothetical proteins, and hypothetical proteins predicted to be cell surface-exposed (based on the presence of a single transmembrane helix motif near the N-terminus of the protein, "Hypothetical external" category) (Fig. 3c; Additional file 2: Table S13). The importance of cell surface modification is apparent in N. acidilobi where $10 \%$ of the proteome is likely involved in glycosylation of the cell surface [16]. Some of the external proteins with high SNP densities may be involved in detecting and binding to hosts or evading host defenses, as shown in other host-symbiont partnerships [75-77], and rapid evolution is frequently observed in these proteins [77, 78]. Notably, even in an insect endosymbiont with extremely low diversity, cell surface proteins are among the genes with the greatest number of SNPs and other variants [78].

\section{Diversification of proteins involved in symbiosis}

Some of the genes with the highest densities of nSNPs may help explain our findings of a potentially broad host range, clonality on a single host, and purifying selection in the overall population. One of these genes is cytochrome bd-I ubiquinol oxidase subunit I (IMG Gene ID 2735310658, Additional file 3), one subunit of a membrane-bound enzyme which transfers electrons from a reduced quinol to $\mathrm{O}_{2}$, generating membrane potential without pumping protons [16, 79, 80]. Subunit II of this enzyme was not annotated by an automated pipeline, but this subunit often has a faster evolutionary rate than subunit I and this divergence can prevent automatic annotation of homologues [81]. Immediately downstream of subunit $\mathrm{I}$, we found a hypothetical integral membrane protein which displays distant similarity ( $25 \%$ amino acid identity) to subunit II. Within subunit I, there was no significant difference in the distribution of nSNPs between different regions (internal, external, transmembrane) $\left(\mathrm{X}^{2}(2, N=453)=0.31574, p=\right.$ 0.854). The functionally important Q-loop responsible for binding with the $\mathrm{O}_{2}$ substrate was conserved except for two nSNPs (Fig. 4), leading us to postulate that it is still a functional enzyme. However, we found neither the ability to synthesize any quinones nor the ability to reduce quinones to quinols, the substrates for this enzyme, within any terrestrial Nanoarchaeota genomes. Nanoarchaeota may be utilizing reduced quinols from the host diffusing through membranes [79] where the cells are connected, and nSNPs in the transmembrane regions of the oxidase might allow them to utilize different quinols if they are associated with different hosts (Fig. 4).

Quinone-mediated energy transfer has been experimentally demonstrated in Geobacter [82], and evidence from 


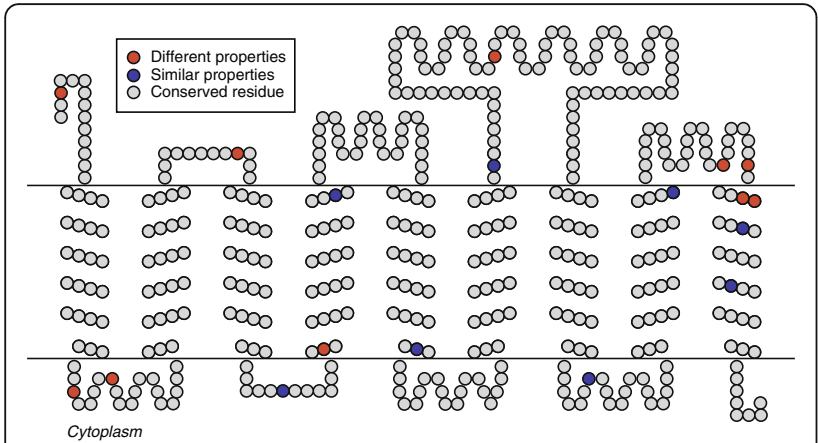

Fig. 4 nSNPs in cytochrome bd-l ubiquinol oxidase in clade 1 Nanoarchaeota. Cartoon of cytochrome bd-I ubiquinol oxidase with residues with $n S N P s$ highlighted, based on the properties of the alternate residue(s) compared to the reference residue in AB-777F03. SSNPs in conserved residues are not shown

other microbe-microbe symbioses suggests this may be a common mechanism for energy exchange. "Chlorochromatium aggregatum" is proposed to exchange quinones between the bacterial partners to create a shared proton motive force to power motility of the central bacterium [75]. Multiple Parcubacteria, also believed to rely at least partially on other microbes, have ubiquinol oxidases but no quinone biosynthesis genes or quinone-dependent $\mathrm{NAD}(\mathrm{P}) \mathrm{H}$ dehydrogenases [76]. N. equitans is an interesting exception from the terrestrial representatives of the phylum in that it possesses a sulfide:quinone oxidoreductase, but not a cytochrome bd-I ubiquinol oxidase. Multi-omics studies have suggested that $N$. equitans may use NADH from $I$. hospitalis with this oxidoreductase to generate ATP [8]. If true, $N$. equitans may be utilizing a similar strategy to that proposed for terrestrial Nanoarchaeota but drawing from a different step on the host electron transport chain. Candidatus Mancarchaeum acidiphilum Mia14 has both a sulfide:quinone oxidoreductase (IMG Gene ID 2758411520) and a cytochrome bd-I ubiquinol oxidase (IMG Gene IDs 2758412059, 2758412060) [22], so it could be utilizing either strategy.

We speculate that quinone-mediated energy transfer between Nanoarchaeota and hosts could lead to clonality on a host. In this proposed scenario, an attached Nanoarchaeota cell would use reduced quinols from the host and thereby slightly deplete the host membrane potential. High membrane potential might be required for successful attachment to a host or to obtain the necessary energy to proliferate on a host. In such a case, other Nanoarchaeota encountering an occupied host cell might fail to initiate or complete attachment due to this reduction in membrane potential. Thus, only the progeny of the first symbiont to attach would proliferate on an individual host cell. Such a process could also apply to $N$. equitans. Unlike other known prokaryotes with two membranes, I. hospitalis has a charged outer membrane due to the localization of ATP synthase in this layer [83], so it would be theoretically possible for $N$. equitans to sense the membrane potential. The clustering of Nanoarchaeota cells on a host cell commonly observed in cultures of both $N$. equitans $[1,5]$ and $N$. acidilobi [16] is consistent with this proposed mechanism. It is also possible that clonality could arise as a consequence of the spatial separation of potential hosts, such that the probability of a given symbiont cell encountering and attaching to a host is low, and the probability of two different symbiont cells attaching to the same host is even lower. Single-cell genomics could be used to determine if clonality is the exception or the rule in other microbe-microbe associations where multiple ectosymbionts are observed attached to a single host. These include TM7 (Saccharibacteria) [25], Candidatus Mancarchaeum acidiphilum Mia14 [22], and phototrophic consortia like "Chlorochromatium aggregatum" [84]. The latter is an especially interesting point of comparison as the ectosymbionts appear to be vertically transmitted when the central cell divides [75].

The second gene with a high density of nSNPs and possible function in the symbiosis is from the "Motility and attachment" functional category. It was annotated as a type 2 secretion system (T2SS) protein F, homologous to FlaJ/ $\mathrm{TadC}$, a membrane platform protein for secretion systems, pili, and flagella [85] (IMG Gene ID 2735310502). There was no significant difference in the distribution of nSNPs between internal, external, and transmembrane regions of the protein $\left(\chi^{2}(2, N=619)=2.2621, p=0.3227\right)$. T2SS have many similarities to type IV pili (T4P) [85] which are abundant and diverse in Archaea [86], so this gene likely encodes part of a T4P system. Genomic, proteomic, and microscopy data indicate that Nanoarchaeota have two different T4P systems as well as a true archaeal flagellum $[16,70,86]$. These appendages likely serve different purposes and can be regulated separately [86]. The flagellum commonly plays a role in motility and generalized attachment to surfaces and other cells [87] and may do the same in Nanoarchaeota and other microbial symbionts [88-90]. Once Nanoarchaeota have formed a weak non-specific attachment to a potential host with the flagellum, the T4P systems might be responsible for sensing the suitability of the host and forming a more secure and specific attachment. Thus, diversification of the T4P systems could be indicative of adaptation to different hosts or compensatory mutations to escape host defenses. The presence of multiple T4P systems broadens this adaptive potential, and interestingly, we found four different FlaJ/TadC homologues with densities of nSNPs from 1.9-37.0 per kb (Additional file 3), suggesting varying levels of evolutionary pressure on these different systems. The importance of T4P and pili in general is apparent by their ubiquity in other microbial symbionts, even those with highly reduced 
genomes and missing many key metabolic functions [22, 23, 76, 91-93].

In this study, single-cell genomics has enabled us to perform a detailed genomic analysis and identify genes that are diversified in a Nanoarchaoeota population and with putative roles in symbiosis. Several of these genes have been repeatedly implicated in host association in other symbioses, lending validity to our approach and conclusions. However, there are important differences in the life history, population diversity, and genomic signatures of selection in Nanoarchaeota compared to microbial endosymbionts of eukaryotes $[34,94]$. This suggests that additional comparison with other microbe-microbe symbioses is needed to clarify which molecular mechanisms underpin these types of associations, what genes and proteins influence host range and host switching, and to what degree are they diverged or conserved. Importantly, Nanoarchaeota are only a single lineage within the DPANN superphylum, many of which are also known or hypothesized to depend on a microbial host [21-23], as are many members of an analogous group of bacteria, the Candidate Phyla Radiation (CPR, or superphylum Patescibacteria) $[92,95]$. Thus, experimentally tractable Nanoarchaeota-host systems may be able to shed light on the molecular mechanisms of microbe-microbe association that could be shared across large swaths of the tree of life.

\section{Conclusions}

In summary, we have demonstrated that Nanoarchaeota can be readily co-sorted with putative hosts in a high-throughput and culture-independent manner using single-cell genomics techniques, enabling us to perform a detailed genomic analysis. Using these data, we have derived a genome-based phylogeny of Nanoarchaeota, defining two species-level clades and suggesting that global diversity remains greatly underexplored. Six novel putative hosts for YNP Nanoarchaeota are proposed, and we suggest that the known host Acd1 "Acidicryptum nanophilum" can associate with multiple species of Nanoarchaeota, broadening the range of possible associations for both hosts and symbionts. Single-cell genomics of co-sorted associations also allowed us to determine that although populations of Nanoarchaeota are diverse, those attached to a single host cell appear to be multiple clonal cells or present as single cells. High overall SNP densities and a low pN/pS imply purifying selection and important differences in evolutionary processes compared to obligate microbial endosymbionts. Genes with high densities of nSNPs included likely cell surface proteins, type IV pili components, and a cytochrome bd-I ubiquinol oxidase, all of which are implicated in interactions with hosts in other microbial symbioses. Based on these genes, we propose a hypothesis for how clonality may be maintained in this symbiosis. Together, these results provide clues about the adaptation of Nanoarchaeota to such a broad range of potential hosts and environmental conditions, providing a new foundation for our understanding of the many other microbe-microbe symbioses thought to exist within the major, yet largely uncultivated branches of the tree of life.

\section{Additional files}

Additional file 1: Figure S1. Map of sampling sites. Figure S2. TNF PCA plots for SAGs illustrating separation of Nanoarchaeota and putative host genome bins. Figure S3. Maximum likelihood phylogeny of phylum Nanoarchaeota based on 165 rRNA gene sequences at least 400 nt in length. Figure S4. Identification of putative host genome bins based on ANI to reference genomes and metagenome bins. Figure S5. Scanning electron micrograph of multiple Nanoarchaeota cells attached to host cells. Figure S6. SNP type and density in individual clade 1 Nanoarchaeota SAGs. Figure S7. Variation in SSNP density in clade 1 Nanoarchaeota genes by functional category. (DOCX $24585 \mathrm{~kb}$ )

Additional file 2: Table S1. Read count, assembly statistics, completeness and contamination estimates from CheckM, and Nanoarchaeota-host pairing information for SAGs and selected Nanoarchaeota reference genomes. Table S2. Data identifiers for SAGs and reference genomes used in this study. Table S3. Assembly statistics, completeness and contamination estimates from CheckM, and additional information for reference genomes for comparison to putative host genome bins. Table S4. Assembly statistics, completeness and contamination estimates from CheckM, and additional information for Nanoarchaeota genome bins $<25 \mathrm{~kb}$, putative host genome bins, and unbinned scaffolds. Table S5. Congruence of $16 \mathrm{~S}$ rRNA gene, ribosomal protein tree, and ANI data for delineating Nanoarchaeota genome bins into clades. Table S6. Average nucleotide identity (ANI) of Nanoarchaeota genome bins and reference genomes. Comparisons $>95 \% \mathrm{ANI}$ are highlighted. All cells with alignment lengths less than $20 \mathrm{~kb}$ have been set to $0 \%$ ANI. Table S7. Similarity of Nanoarchaeota 165 rRNA gene sequences extracted from genome bins and references. Sequences that are $>98 \%$ similar are highlighted in green, sequence lengths are shown in parentheses. Table S8. Average nucleotide identity (ANI) of putative host genome bins and host reference genomes. Comparisons $>95 \% \mathrm{ANI}$ are highlighted. All cells with alignment lengths less than $20 \mathrm{~kb}$ have been set to 0\% ANI. Table S9. Alignment results for proteins potentially horizontally transferred between hosts and

Nanoarchaeota. Table S10. Within-SAG SNPs in single-sorted Nanoarchaeota SAGs and co-sorted Nanoarchaeota and putative host genome bins, at a MAF $>10 \%$. Table S11. Simulated within-SAG SNPs for pooled datasets of 1 to 6 SAGs, at a MAF > 10\%. Table S12. SNPs in all clade 1 Nanoarchaeota genome bins (> $100 \mathrm{~kb}$ bin size), mean values shown for each site type. Table S13. Pairwise comparisons of mean non-synonymous SNPs per kb between functional categories of genes in clade 1 Nanoarchaeota SAGs and SAG bins, from post-hoc Tukey HSD tests. Significant comparisons are highlighted in green. (XLSX $65 \mathrm{~kb}$ )

Additional file 3: SSNP and nSNP data for genes in clade 1 Nanoarchaeota SAGs and genome bins, organized in tabs by functional category. Genes highlighted in the discussion are separated into their own tab and highlighted in green within their respective functional category tabs. (XLSX $119 \mathrm{~kb})$

\section{Abbreviations}

ANI: Average nucleotide identity; DPANN: Superphylum of Archaea originally comprising Diapherotrites, Parvarchaeota, Aenigmarchaeota, Nanohaloarchaeota, and Nanoarchaeota, now including also Woesearchaeota, Pacearchaeota, and Micrarchaeota; MAF: Minor allele frequency; MDA: Multiple displacement amplification; ML: Maximum likelihood; nSNP: Non-synonymous SNP; PCA: Principal component analysis; $\mathrm{pN} / \mathrm{pS}$ : Ratio of non-synonymous SNP/non-synonymous site to synonymous SNP/synonymous site; RP: Ribosomal protein; SAG: Single amplified genome; SNP: Single nucleotide polymorphism; sSNP: Synonymous SNP;

TNF: Tetranucelotide frequency; YNP: Yellowstone National Park 


\section{Acknowledgements}

We would like to thank Grant Jensen and Davi Ortega at the California Institute of Technology for their efforts to acquire electron microscopy images from challenging environmental samples and Paul Oikawa for assistance with editing figures in Illustrator.

\section{Funding}

The work conducted by the U.S. Department of Energy Joint Genome Institute, a DOE Office of Science User Facility, is supported under Contract No. DE-AC02-05CH11231. The work was also supported by the NSF grants DEB-1441717 and OCe-1335810 to R.S.

\section{Availability of data and materials}

Reads and most reference genomes used in this study are accessible through NCBI with the identifiers listed in Additional file 2: Table S2. Nanoarchaeota SAGs, genome bins, additional reference genomes, supporting files for $16 \mathrm{~S}$ rRNA gene and ribosomal protein phylogenies, scripts and output data from SNP analysis, and amino acid alignments of featured genes involved in symbiosis are available from the "Nanoarchaeota co-sorts" Bitbucket repo at [https://bitbucket.org/jessica_jarett/nanoarchaeota-co-sorts/].

\section{Authors' contributions}

WI, JM-M, MY, ZJJ, and JPB collected and processed samples. RM and RS generated SAGs. SN generated SNP data. NNI provided conceptual advice on functional analysis of SNP data. FS generated the ribosomal protein phylogeny and provided assistance on $16 \mathrm{~S}$ rRNA phylogeny. JKJ analyzed and visualized all other data. MP, NCK and WI provided subject area expertise. TW supervised research. JKJ and TW prepared the manuscript, with contributions from all authors. All authors read and approved the final manuscript.

\section{Ethics approval and consent to participate}

Not applicable.

\section{Consent for publication}

Not applicable.

\section{Competing interests}

The authors declare that they have no competing interests.

\section{Publisher's Note}

Springer Nature remains neutral with regard to jurisdictional claims in published maps and institutional affiliations.

\section{Author details}

'DOE Joint Genome Institute, Walnut Creek, CA, USA. ${ }^{2}$ Oak Ridge National Laboratory, Oak Ridge, TN, USA. ${ }^{3}$ Department of Microbiology, University of Tennessee, Knoxville, TN, USA. ${ }^{4}$ Department of Land Resources and Environmental Sciences, Montana State University, Bozeman, MT, USA. ${ }^{5}$ Department of Microbiology and Immunology, Montana State University, Bozeman, MT, USA. ${ }^{6}$ Department of Plant Sciences and Plant Pathology, Montana State University, Bozeman, MT, USA. ${ }^{7}$ Bigelow Laboratory for Ocean Sciences, East Boothbay, ME, USA.

Received: 19 June 2018 Accepted: 26 August 2018

Published online: 17 September 2018

\section{References}

1. Huber H, Hohn MJ, Rachel R, Fuchs T, Wimmer VC, Stetter KO. A new phylum of Archaea represented by a nanosized hyperthermophilic symbiont. Nature. 2002;417:63-7.

2. Heimerl T, Flechsler J, Pickl C, Heinz V, Salecker B, Zweck J, et al. A complex endomembrane system in the Archaeon Ignicoccus hospitalis tapped by Nanoarchaeum equitans. Front Microbiol. 2017:8:1072.

3. Waters E, Hohn MJ, Ahel I, Graham DE, Adams MD, Barnstead M, et al. The genome of Nanoarchaeum equitans: insights into early archaeal evolution and derived parasitism. Proc Natl Acad Sci U S A. 2003;100: 12984-8.

4. Giannone RJ, Huber $H$, Karpinets T, Heimerl T, Küper U, Rachel R, et al. Proteomic characterization of cellular and molecular processes that enable the Nanoarchaeum equitans--Ignicoccus hospitalis relationship. PLoS One. 2011;6:e22942.

5. Jahn U, Gallenberger M, Paper W, Junglas B, Eisenreich W, Stetter KO, et al. Nanoarchaeum equitans and Ignicoccus hospitalis: new insights into a unique, intimate association of two archaea. J Bacteriol. 2008;190:1743-50.

6. Giannone RJ, Wurch LL, Heimerl T, Martin S, Yang Z, Huber H, et al. Life on the edge: functional genomic response of Ignicoccus hospitalis to the presence of Nanoarchaeum equitans. ISME J. 2015;9:101-14.

7. Podar M, Anderson I, Makarova KS, Elkins JG, Ivanova N, Wall MA, et al. A genomic analysis of the archaeal system Ignicoccus hospitalisNanoarchaeum equitans. Genome Biol. 2008;9:R158.

8. Rawle RA, Hamerly T, Tripet BP, Giannone RJ, Wurch L, Hettich RL, et al. Multiomics analysis provides insight to the Ignicoccus hospitalis-Nanoarchaeum equitans association. Biochim Biophys Acta. 2017;1861:2218-27.

9. Junglas B, Briegel A, Burghardt T, Walther P, Wirth R, Huber $\mathrm{H}$, et al. Ignicoccus hospitalis and Nanoarchaeum equitans: ultrastructure, cell-cell interaction, and 3D reconstruction from serial sections of freeze-substituted cells and by electron cryotomography. Arch Microbiol. 2008;190:395-408.

10. Hamerly T, Tripet BP, Tigges M, Giannone RJ, Wurch L, Hettich RL, et al. Untargeted metabolomics studies employing NMR and LC-MS reveal metabolic coupling between Nanoarcheum equitans and its archaeal host Ignicoccus hospitalis. Metabolomics. 2014;11:895-907.

11. Casanueva A, Galada N, Baker GC, Grant WD, Heaphy S, Jones B, et al. Nanoarchaeal 165 rRNA gene sequences are widely dispersed in hyperthermophilic and mesophilic halophilic environments. Extremophiles. 2008;12:651-6.

12. Mardanov AV, Gumerov VM, Beletsky AV, Ravin NV. Microbial diversity in acidic thermal pools in the Uzon Caldera, Kamchatka. Antonie Van Leeuwenhoek. 2018;111:35-43.

13. McCliment EA, Voglesonger KM, O'Day PA, Dunn EE, Holloway JR, Craig Cary S. Colonization of nascent, deep-sea hydrothermal vents by a novel Archaeal and Nanoarchaeal assemblage. Environ Microbiol. 2006;8:114-25.

14. Hohn MJ, Hedlund BP, Huber H. Detection of 165 rDNA sequences representing the novel phylum "Nanoarchaeota": indication for a wide distribution in high temperature biotopes. Syst Appl Microbiol. 2002;25:551-4.

15. Clingenpeel S, Kan J, Macur RE, Woyke T, Lovalvo D, Varley J, et al. Yellowstone lake nanoarchaeota. Front Microbiol. 2013;4:274.

16. Wurch L, Giannone R, Belisle BS, Swift C, Utturkar S, Hettich RL, et al. Genomicsinformed isolation and characterization of a symbiotic Nanoarchaeota system from a terrestrial geothermal environment. Nat Commun. 2016;7:12115.

17. Podar M, Makarova KS, Graham DE, Wolf YI, Koonin EV, Reysenbach A-L. Insights into archaeal evolution and symbiosis from the genomes of a nanoarchaeon and its inferred crenarchaeal host from Obsidian Pool, Yellowstone National Park. Biol Direct. 2013:8:9.

18. Rinke C, Schwientek P, Sczyrba A, Ivanova NN, Anderson IJ, Cheng J-F, et al. Insights into the phylogeny and coding potential of microbial dark matter. Nature. 2013:499:431-7.

19. Williams TA, Szöllősi GJ, Spang A, Foster PG, Heaps SE, Boussau B, et al. Integrative modeling of gene and genome evolution roots the archaeal tree of life. Proc Natl Acad Sci U S A. 2017;114:E4602-11.

20. Adam PS, Borrel G, Brochier-Armanet C, Gribaldo S. The growing tree of Archaea: new perspectives on their diversity, evolution and ecology. ISME J. 2017:11:2407-25.

21. Castelle CJ, Wrighton KC, Thomas BC, Hug LA, Brown CT, Wilkins MJ, et al. Genomic expansion of domain archaea highlights roles for organisms from new phyla in anaerobic carbon cycling. Curr Biol. 2015;25:690-701.

22. Golyshina OV, Toshchakov SV, Makarova KS, Gavrilov SN, Korzhenkov AA, La Cono V, et al. "ARMAN" archaea depend on association with euryarchaeal host in culture and in situ. Nat Commun. 2017;8:60.

23. Comolli LR, Banfield JF. Inter-species interconnections in acid mine drainage microbial communities. Front Microbiol. 2014;5:367.

24. Merkel AY, Pimenov NV, Rusanov II, Slobodkin Al, Slobodkina GB, Tarnovetckii IY, et al. Microbial diversity and autotrophic activity in Kamchatka hot springs. Extremophiles. 2017;21:307-17.

25. He X, McLean JS, Edlund A, Yooseph S, Hall AP, Liu S-Y, et al. Cultivation of a human-associated TM7 phylotype reveals a reduced genome and epibiotic parasitic lifestyle. Proc Natl Acad Sci U S A. 2015;112:244-9.

26. McCutcheon JP. Moran NA. Extreme genome reduction in symbiotic bacteria. Nat Rev Microbiol. 2011;10:13-26.

27. Nicks T, Rahn-Lee L. Inside out: Archaeal ectosymbionts suggest a second model of reduced-genome evolution. Front Microbiol. 2017;8:384. 
28. Munson-McGee JH, Field EK, Bateson M, Rooney C, Stepanauskas R, Young MJ. Nanoarchaeota, their sulfolobales host, and nanoarchaeota virus distribution across Yellowstone National Park Hot Springs. Appl Environ Microbiol. 2015;81:7860-8.

29. Munson-McGee JH, Peng S, Dewerff S, Stepanauskas R, Whitaker RJ, Weitz JS, et al. A virus or more in (nearly) every cell: ubiquitous networks of virus-host interactions in extreme environments. ISME J. 2018;12:1706-14.

30. Randau L. RNA processing in the minimal organism Nanoarchaeum equitans. Genome Biol. 2012;13:R63.

31. Mira A, Moran NA. Estimating population size and transmission bottlenecks in maternally transmitted endosymbiotic bacteria. Microb Ecol. 2002:44:137-43.

32. Funk DJ, Wernegreen JJ, Moran NA. Intraspecific variation in symbiont genomes: bottlenecks and the aphid-buchnera association. Genetics. 2001;157:477-89.

33. Woolfit M, Bromham L. Increased rates of sequence evolution in endosymbiotic bacteria and fungi with small effective population sizes. Mol Biol Evol. 2003:20:1545-55.

34. Wernegreen JJ. Genome evolution in bacterial endosymbionts of insects. Nat Rev Genet. 2002;3:850-61.

35. Latorre A, Manzano-Marín A. Dissecting genome reduction and trait loss in insect endosymbionts. Ann N Y Acad Sci. 2017;1389:52-75.

36. Lieberman TD, Flett KB, Yelin I, Martin TR, McAdam AJ, Priebe GP, et al. Genetic variation of a bacterial pathogen within individuals with cystic fibrosis provides a record of selective pressures. Nat Genet. 2014;46:82-7.

37. Bendall ML, Stevens SL, Chan L-K, Malfatti S, Schwientek P, Tremblay J, et al. Genome-wide selective sweeps and gene-specific sweeps in natural bacterial populations. ISME J. 2016;10:1589-601.

38. Woyke T, Doud DFR, Schulz F. The trajectory of microbial single-cell sequencing. Nat Methods. 2017;14:1045-54.

39. Inskeep W. The YNP metagenome project: environmental parameters responsible for microbial distribution in the Yellowstone geothermal ecosystem. Front Microbiol. 2013;4:67.

40. Rinke C, Lee J, Nath N, Goudeau D, Thompson B, Poulton N, et al. Obtaining genomes from uncultivated environmental microorganisms using FACSbased single-cell genomics. Nat Protoc. 2014;9:1038-48.

41. Bushnell B. BBTools package. https://jgi.doe.gov/data-and-tools/bbtools/. Accessed 1 June 2018.

42. Nurk S, Bankevich A, Antipov D, Gurevich AA, Korobeynikov A, Lapidus A, et al. Assembling single-cell genomes and mini-metagenomes from chimeric MDA products. J Comput Biol. 2013;20:714-37.

43. Kang DD, Froula J, Egan R, Wang Z. MetaBAT, an efficient tool for accurately reconstructing single genomes from complex microbial communities. PeerJ. 2015;3:e1165.

44. Eren AM, Murat Eren A, Esen ÖC, Quince C, Vineis JH, Morrison HG, et al. Anvi'o: an advanced analysis and visualization platform for 'omics data. PeerJ. 2015;3:e1319.

45. Altschul SF, Gish W, Miller W, Myers EW, Lipman DJ. Basic local alignment search tool. J Mol Biol. 1990;215:403-10.

46. Parks DH, Imelfort M, Skennerton CT, Hugenholtz P, Tyson GW. CheckM: assessing the quality of microbial genomes recovered from isolates, single cells, and metagenomes. Genome Res. 2015;25:1043-55.

47. Pritchard L, Glover RH, Humphris S, Elphinstone JG, Toth IK. Genomics and taxonomy in diagnostics for food security: soft-rotting enterobacterial plant pathogens. Anal Methods. 2016;8:12-24.

48. Richter M, Rosselló-Móra R. Shifting the genomic gold standard for the prokaryotic species definition. Proc Natl Acad Sci U S A. 2009;106:19126-31.

49. Barter R, Yu B. superheat: a graphical tool for exploring complex datasets using heatmaps. R package version 0.1.0. https://github.com/rlbarter/ superheat. Accessed 1 June 2018.

50. Konstantinidis KT, Rosselló-Móra R, Amann R. Uncultivated microbes in need of their own taxonomy. ISME J. 2017;11:2399-406.

51. Yu FB, Blainey PC, Schulz F, Woyke T, Horowitz MA, Quake SR. Microfluidic-based mini-metagenomics enables discovery of novel microbial lineages from complex environmental samples. Elife. 2017;6. https://doi.org/10.7554/eLife.26580.

52. Eddy SR. Accelerated profile HMM searches. PLoS Comput Biol. 2011;7:e1002195.

53. Lartillot N, Rodrigue N, Stubbs D, Richer J. PhyloBayes MPI: phylogenetic reconstruction with infinite mixtures of profiles in a parallel environment. Syst Biol. 2013;62:611-5.
54. Yu G, Smith DK, Zhu H, Guan Y, TT-Y L. ggtree: an r package for visualization and annotation of phylogenetic trees with their covariates and other associated data. Methods Ecol Evol. 2016;8:28-36.

55. Waterhouse AM, Procter JB, Martin DMA, Clamp M, Barton GJ. Jalview Version 2--a multiple sequence alignment editor and analysis workbench. Bioinformatics. 2009:25:1189-91.

56. Nawrocki EP. Structural RNA homology search and alignment using covariance models. All Theses and Dissertations. 2009:256.

57. Quast C, Pruesse E, Yilmaz P, Gerken J, Schweer T, Yarza P, et al. The SILVA ribosomal RNA gene database project: improved data processing and webbased tools. Nucleic Acids Res. 2013:41(Database issue):D590-6.

58. Yilmaz P, Parfrey LW, Yarza P, Gerken J, Pruesse E, Quast C, et al. The SILVA and "All-species Living Tree Project (LTP)" taxonomic frameworks. Nucleic Acids Res. 2013;42:D643-8.

59. Nguyen L-T, Schmidt HA, von Haeseler A, Minh BQ. IQ-TREE: a fast and effective stochastic algorithm for estimating maximum-likelihood phylogenies. Mol Biol Evol. 2015;32:268-74.

60. Kalyaanamoorthy $S$, Minh BQ, Wong TKF, von Haeseler A, Jermiin LS. ModelFinder: fast model selection for accurate phylogenetic estimates. Nat Methods. 2017;14:587-9.

61. Bojanowski M, Edwards R. alluvial: R package for creating alluvial diagrams. R package version: 0.2-0. 2017. https://github.com/mbojan/ alluvial. Accessed 1 June 2018.

62. Buchfink B, Xie C, Huson DH. Fast and sensitive protein alignment using DIAMOND. Nat Methods. 2015;12:59-60.

63. Nayfach S, Rodriguez-Mueller B, Garud N, Pollard KS. An integrated metagenomics pipeline for strain profiling reveals novel patterns of bacterial transmission and biogeography. Genome Res. 2016;26:1612-25.

64. Clingenpeel S, Clum A, Schwientek P, Rinke C, Woyke T. Reconstructing each cell's genome within complex microbial communities - dream or reality? Front Microbiol. 2015;5:771

65. Bowers RM, Kyrpides NC, Stepanauskas R, Harmon-Smith M, Doud D, Reddy TBK, et al. Minimum information about a single amplified genome (MISAG) and a metagenome-assembled genome (MIMAG) of bacteria and archaea. Nat Biotechnol. 2017;35:725-31.

66. Altamia MA, Wood N, Fung JM, Dedrick S, Linton EW, Concepcion GP, et al. Genetic differentiation among isolates of Teredinibacter turnerae, a widely occurring intracellular endosymbiont of shipworms. Mol Ecol. 2014;23:1418-32.

67. Engel P, Stepanauskas R, Moran NA. Hidden diversity in honey bee gut symbionts detected by single-cell genomics. PLoS Genet. 2014;10:e1004596.

68. Veith A, Klingl A, Zolghadr B, Lauber K, Mentele R, Lottspeich F, et al. Acidianus, Sulfolobus and Metallosphaera surface layers: structure composition and gene expression. Mol Microbiol. 2009;73:58-72.

69. Claus H, Akça E, Debaerdemaeker T, Evrard C, Declercq J-P, König H. Primary structure of selected archaeal mesophilic and extremely thermophilic outer surface layer proteins. Syst Appl Microbiol. 2002;25:3-12.

70. Huber H, Hohn MJ, Stetter KO, Rachel R. The phylum Nanoarchaeota: present knowledge and future perspectives of a unique form of life. Res Microbiol. 2003;154:165-71

71. Kashtan N, Roggensack SE, Rodrigue S, Thompson JW, Biller SJ, Coe A, et al. Single-cell genomics reveals hundreds of coexisting subpopulations in wild Prochlorococcus. Science. 2014:344:416-20.

72. Moran NA. Accelerated evolution and Muller's rachet in endosymbiotic bacteria. Proc Natl Acad Sci. 1996;93:2873-8.

73. Clark MA, Moran NA, Baumann P. Sequence evolution in bacterial endosymbionts having extreme base compositions. Mol Biol Evol. 1999;16:1586-98.

74. Tamas I, Klasson L, Canbäck B, Näslund AK, Eriksson A-S, Wernegreen JJ, et al. 50 million years of genomic stasis in endosymbiotic bacteria. Science. 2002;296:2376-9.

75. Liu Z, Müller J, Li T, Alvey RM, Vogl K, Frigaard N-U, et al. Genomic analysis reveals key aspects of prokaryotic symbiosis in the phototrophic consortium "Chlorochromatium aggregatum". Genome Biol. 2013;14:R127.

76. Nelson WC, Stegen JC. The reduced genomes of Parcubacteria (OD1) contain signatures of a symbiotic lifestyle. Front Microbiol. 2015:6:713.

77. Paul BG, Burstein D, Castelle CJ, Handa S, Arambula D, Czornyj E, et al. Retroelement-guided protein diversification abounds in vast lineages of Bacteria and Archaea. Nat Microbiol. 2017;2:17045.

78. Woyke T, Tighe D, Mavromatis K, Clum A, Copeland A, Schackwitz W, et al. One bacterial cell. one complete genome. PLoS One. 2010;5:e10314. 
79. Borisov VB, Gennis RB, Hemp J, Verkhovsky MI. The cytochrome bd respiratory oxygen reductases. Biochim Biophys Acta. 2011;1807: 1398-413.

80. Simon J, van Spanning RJM, Richardson DJ. The organisation of proton motive and non-proton motive redox loops in prokaryotic respiratory systems. Biochim Biophys Acta. 2008;1777:1480-90.

81. Hao W, Golding GB. Asymmetrical evolution of cytochrome bd subunits. J Mol Evol. 2006;62:132-42.

82. Smith JA, Nevin KP, Lovley DR. Syntrophic growth via quinone-mediated interspecies electron transfer. Front Microbiol. 2015;6:121.

83. Küper U, Meyer C, Müller V, Rachel R, Huber H. Energized outer membrane and spatial separation of metabolic processes in the hyperthermophilic Archaeon Ignicoccus hospitalis. Proc Natl Acad Sci U S A. 2010;107:3152-6.

84. Overmann J, Schubert K. Phototrophic consortia: model systems for symbiotic interrelations between prokaryotes. Arch Microbiol. 2002;177:201-8.

85. Peabody CR, Chung YJ, Yen M-R, Vidal-Ingigliardi D, Pugsley AP, Saier MH Jr. Type II protein secretion and its relationship to bacterial type IV pili and archaeal flagella. Microbiology. 2003;149(Pt 11):3051-72.

86. Makarova KS, Koonin EV, Albers S-V. Diversity and evolution of type IV pili systems in Archaea. Front Microbiol. 2016;7:667.

87. Näther DJ, Rachel R, Wanner G, Wirth R. Flagella of Pyrococcus furiosus: multifunctional organelles, made for swimming, adhesion to various surfaces, and cell-cell contacts. J Bacteriol. 2006;188:6915-23.

88. Akman L, Yamashita A, Watanabe H, Oshima K, Shiba T, Hattori M, et al. Genome sequence of the endocellular obligate symbiont of tsetse flies, Wigglesworthia glossinidia. Nat Genet. 2002;32:402-7.

89. Rio RVM, Symula RE, Wang J, Lohs C, Wu Y-N, Snyder AK, et al. Insight into the transmission biology and species-specific functional capabilities of tsetse (Diptera: Glossinidae) Obligate Symbiont Wigglesworthia. MBio. 2012; 3:e00240-11.

90. Collingro A, Köstlbacher S, Mussmann M, Stepanauskas R, Hallam SJ, Horn M. Unexpected genomic features in widespread intracellular bacteria: evidence for motility of marine chlamydiae. ISME J. 2017;11: 2334-44.

91. Yeoh YK, Sekiguchi Y, Parks DH, Hugenholtz P. Comparative genomics of candidate phylum TM6 suggests that parasitism is widespread and ancestral in this lineage. Mol Biol Evol. 2016;33:915-27.

92. Luef B, Frischkorn KR, Wrighton KC, Holman H-YN, Birarda G, Thomas $B C$, et al. Diverse uncultivated ultra-small bacterial cells in groundwater. Nat Commun. 2015;6:6372.

93. Gong J, Qing Y, Guo X, Warren A. "Candidatus Sonnebornia yantaiensis", a member of candidate division OD1, as intracellular bacteria of the ciliated protist Paramecium bursaria (Ciliophora, Oligohymenophorea) Syst Appl Microbiol. 2014;37:35-41.

94. Bennett GM, Moran NA. Heritable symbiosis: the advantages and perils of an evolutionary rabbit hole. Proc Natl Acad Sci U S A. 2015;112:10169-76.

95. Brown CT, Hug LA, Thomas BC, Sharon I, Castelle CJ, Singh A, et al. Unusual biology across a group comprising more than $15 \%$ of domain bacteria. Nature. 2015:523:208-11.

Ready to submit your research? Choose BMC and benefit from:

- fast, convenient online submission

- thorough peer review by experienced researchers in your field

- rapid publication on acceptance

- support for research data, including large and complex data types

- gold Open Access which fosters wider collaboration and increased citations

- maximum visibility for your research: over $100 \mathrm{M}$ website views per year

At BMC, research is always in progress.

Learn more biomedcentral.com/submissions 